Research

\title{
Precision of fiducial marker alignment for correlative super-resolution fluorescence and transmission electron microscopy
}

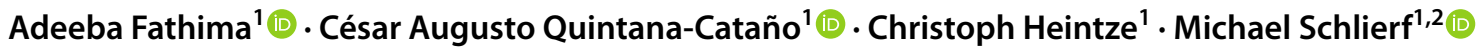

Received: 22 December 2020 / Accepted: 24 March 2021

(c) The Author(s) $2021 \quad$ OPEN

\begin{abstract}
Recent advances in microscopy techniques enabled nanoscale discoveries in biology. In particular, electron microscopy reveals important cellular structures with nanometer resolution, yet it is hard, and sometimes impossible to resolve specific protein localizations. Super-resolution fluorescence microscopy techniques developed over the recent years allow for protein-specific localization with $\sim 20 \mathrm{~nm}$ precision are overcoming this limitation, yet it remains challenging to place those in cells without a reference frame. Correlative light and electron microscopy (CLEM) approaches have been developed to place the fluorescence image in the context of a cellular structure. However, combining imaging methods such as super resolution microscopy and transmission electron microscopy necessitates a correlation using fiducial markers to locate the fluorescence on the structures visible in electron microscopy, with a measurable precision. Here, we investigated different fiducial markers for super-resolution CLEM (sCLEM) by evaluating their shape, intensity, stability and compatibility with photoactivatable fluorescent proteins as well as the electron density. We further carefully determined limitations of correlation accuracy. We found that spectrally-shifted FluoSpheres are well suited as fiducial markers for correlating single-molecule localization microscopy with transmission electron microscopy.
\end{abstract}

\section{Introduction}

Fluorescence microscopy has facilitated numerous discoveries in cell biology over the past decades. While being incredibly powerful, in particular with fluorescent protein fusions, the resolution remained diffraction-limited at $\sim 250 \mathrm{~nm}$, which is much larger than most molecular arrangements within the cells. On the other hand, electron microscopy (EM) has historically showcased (sub)nanometer resolution limits, however with the drawback that the samples must be dehydrated and that the electron density contrast is mostly non-specific to proteins. Already early-on, the non-specificity was addressed with immunogold electron microscopy, which depends on a good antibody accessibility. To overcome this limitation, the introduction of correlative fluorescence light and electron microscopy imaging (CLEM) merged the advantages of both distinct methods [1, 2]. Yet, typical resolutions of fluorescence microscopes imposed a vast mismatch with nanometer-resolved electron microscopy data. The advent of superresolution fluorescence microscopy has been making revolutionary strides in biological imaging [3]. Single molecule localization (SMLM) super-resolution methods such as PALM [4] and (d)STORM [5, 6] achieve localization accuracies on the order of 10-20 nm. Thus, correlating superresolution fluorescence and electron microscopy (sCLEM) data allows for a more accurate positioning of each molecule to its structural location within in the cellular context.

Supplementary Information The online version contains supplementary material available at https://doi.org/10.1007/s43939-02100011-1.

$\triangle$ Michael Schlierf, michael.schlierf@tu-dresden.de | 1 B CUBE - Center for Molecular Bioengineering, TU Dresden, Tatzberg 41, 01307 Dresden, Germany. ${ }^{2}$ Cluster of Excellence Physics of Life, TU Dresden, 01062 Dresden, Germany.

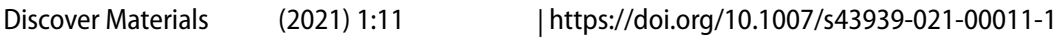

Published online: 08 April 2021 
Correlation of images from two methods is typically based on landmarks, e.g. recognizable cellular structures from the specimen itself or specialized materials introduced as fiducial markers [7, 8]. However, cellular landmarks mandate previously known or discernible protein patterns reminiscent of the physical structure visualized in EM. In the case of unknown cellular localization, the use of additional fiducial markers is a prerequisite. An ideal fiducial marker is an object, which is visible in both imaging methods to register the two images preferably with an ascertainable precision and accuracy, and thus has to fulfill certain criteria (Table 1). For fluorescence imaging, the emission wavelength of the fiducial marker should be comparable with the fluorophore of interest to avoid chromatic aberration corrections during post-processing. The fluorescence intensity of the fiducial marker should not be too bright to avoid detector saturation or too dim to avoid a low localization accuracy. For transmission electron microscopy, the fiducial marker should present a high electron density to be detectable in TEM $[9,10]$. Various fiducial markers have been employed in the past years, including polymer fluorescent beads $[7,11]$, gold nanoparticles $[12,13]$ or quantum dots $[9,14,15]$. Polymer fluorescent beads show a good electron contrast. However, if their emission spectrum directly overlaps with the spectrum of the fluorophore of interest, they are typically too bright at the high detector gain settings required for single-molecule localization microscopy. Washing with Tween-20 or pre-bleaching, however, led to optical non-uniformity which reduces the localization accuracy $[7,16]$. Balinovic et al. recently suggested the use of spectrally shifted polymer fluorescent beads, to overcome the intensity differences for single-molecule localization microscopy [16]. Other correlative studies used gold nanoparticles or quantum dots as fiducial markers [13]. However, an assessment of different fiducial markers for correlative SMLM and TEM imaging is currently lacking. In this study, we compare fluorescent gold nanoparticles (AuNP), quantum dot beads (QB) and FluoSpheres (FS) for their characteristics suitable in both imaging methods (Table 1). We evaluated their shape, intensity, stability, and compatibility with a typical photoactivatable fluorescent protein as well as their electron density. We further discuss correlation accuracy and provide an algorithm to prevent under-estimating the correlation accuracy. We finally demonstrate the correlation accuracy with a biosilica-associated protein fused to Dendra2 and located in the valve of the diatom Thalassiosira pseudonana.

\section{Results and discussions}

Many photoconvertible fluorescent proteins emit in the range of 570-640 nm [17]. Therefore, we determined the emission spectra of fiducial markers and used those that partially overlap with the emission the photoconvertible protein Dendra2 (Fig. 1a). Subsequently, we immobilized gold nanoparticles (AuNP), Quantum Dot Beads (QB) and FluoroSpheres (FS) on a carbon-coated TEM grid, which will be later used for subsequent TEM imaging. The carbon-coated TEM grid was placed on a glass coverslip for fluorescence microscopy and imaged in near-TIRF conditions, following a highly-inclined illumination scheme [18] (Fig. 1b). All three fiducial markers showed a nearly diffraction-limited image, though with very different fluorescence intensities. Surprisingly, the AuNPs showed despite the largest emission spectrum overlap (Fig. 1a) the lowest signal intensity of 150-200 photon counts per frame, while both, the QBs and the FSs showed bright intensities of typically 700-870 photon counts per frame (insets in Fig. 1b). QBs showed an inhomogeneous intensity distribution among different fiducials ranging from 400 to 800 photon counts per frame (Fig. 1C), likely due to their nature of clustering of quantum dots to form quantum beads. The selected FSs showed a strongly shifted emission spectrum (Fig. 1a), nevertheless to our surprise, their emission signal was stable and bright. For comparison, single Dendra2 fluorophores showed typically a maximum intensity of $\sim 180$ photon counts per frame.

While the brightness of the fiducial is important for an unambiguous identification, an automated analysis based on single-molecule localization typically requires a high uniformity in shape. We determined the uniformity of AuNPs, QBs and FSs (Fig. 1d) and found that FSs presented very good circularity $(0.95 \pm 0.15$ (mean \pm s.d.), $n=194)$ and the most homogenous area $\left(0.25 \pm 0.23 \mu \mathrm{m}^{2}\right.$, median: $\left.0.18 \mu \mathrm{m}^{2}\right)$. AuNPs showed poorer circularity $(0.65 \pm 0.32, \mathrm{n}=83)$,

Table 1 Characteristics of fiducial markers for sCLEM

\begin{tabular}{ll}
\hline Characteristic & Desirable quality \\
\hline Emission wavelengths & Visible in the same emission channel as the fluorophore of interest \\
Fluorescence intensity & Bright, but avoid detector saturation \\
Localization uncertainty & Stable emission over the duration of PALM/STORM data acquisition \\
Shape profile & Circular and non-aggregating, homogenous size distribution \\
Electron density & Strong contrast in EM without external enhancement \\
\hline
\end{tabular}



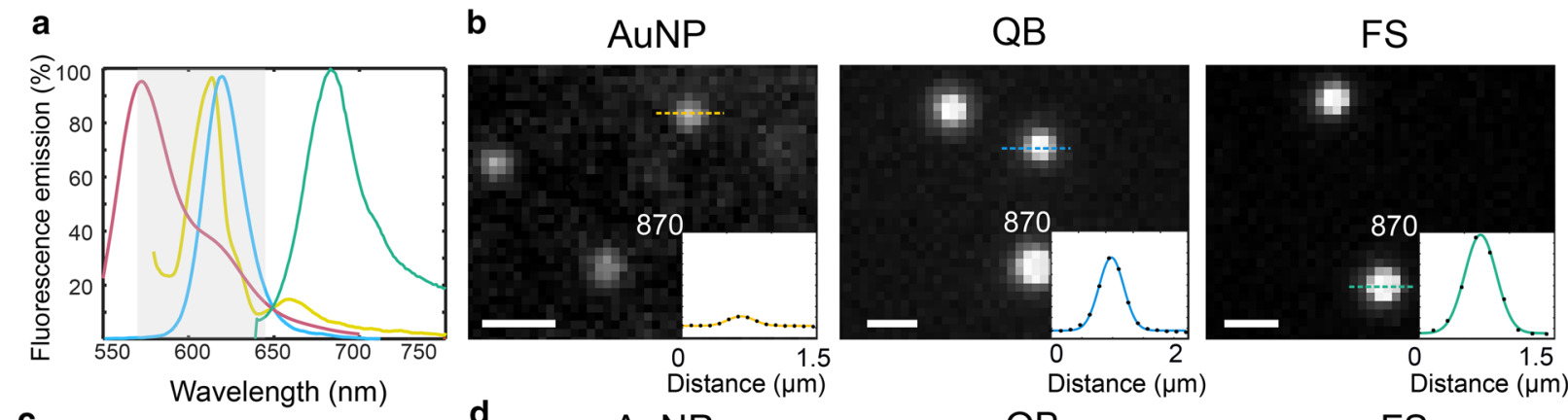

C
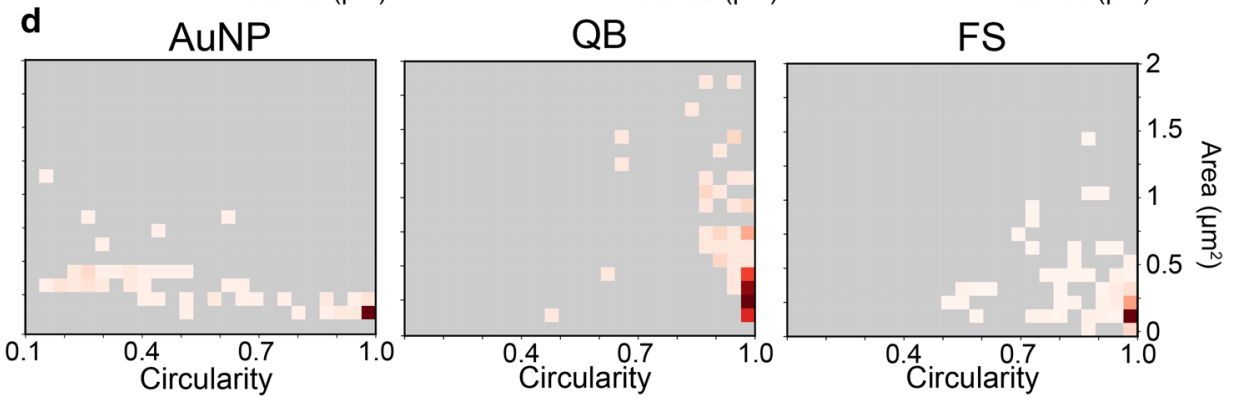

Fig. 1 Fluorescence profile of different fiducials. a Emission spectrum of Gold nanoparticles (yellow), Quantum Beads (blue), dark red FluoSpheres (green) and Dendra2 (red). Gray highlight corresponds to the emission filter bandwidth in fluorescence microscopy $\mathbf{b}$ Fluorescence images of typical AuNPs, QBs and FSs. Insets: Intensity profile of the corresponding fiducial marker. c Maximum intensity distributions of FSs, AuNPs and QBs. d Shape descriptors of AuNPs, QBs and FSs showing circularity and apparent area

suggesting aggregate formation before immobilization. QBs exhibited a high circularity $(0.94 \pm 0.09, \mathrm{n}=81)$, but a large variation in area $\left(0.63 \pm 0.54 \mu \mathrm{m}^{2}\right.$, median: $\left.0.44 \mu \mathrm{m}^{2}\right)$, which could be the result of their nature as being formed of an agglomerate of quantum dots.

SMLM images are reconstructed from hundreds of individual frames with scarce fluorophore densities. To obtain a high confidence reconstructed image, it is important to minimize any nanoscale drift during imaging over tens of seconds. Physical means for drift reduction are often not sufficient and therefore fiducial marker positions are tracked and used to correct any frame-to-frame drift [16]. This necessitates that fiducial markers are photostable, with a reliable emission intensity during imaging over hundreds of frames. Thus, we compared AuNPs, QBs and FSs emission for 2000 frames (60 ms exposure time) (Fig. 2a). Surprisingly, AuNPs were very unstable and barely detectable beyond $\sim 400$ frames. Some QBs showed a stable emission intensity, while others showed large fluctuations in intensity, potentially originating from their quantum dot composition, which are known to blink [19]. FSs appeared consistently photostable and only rarely decreased in their emission intensity over 2000 frames. The low stability of the AuNPs did not allow any further analysis of long-term stability. We subsequently determined the localization accuracy of QBs and FSs for each frame and tracked their X and Y drift during the course of 2000 frames (120 s) and used ThunderSTORM [20] to correct for the detected drift (Fig. 2b). Both, QBs and FSs allowed for a stable drift correction, though the intensity fluctuating QBs showed a less reliable localization accuracy and drift correction (Fig. 2c). To evaluate the positional stability and reliability, we determined the localization accuracy standard deviation of individual fiducial markers for 50 frame intervals after drift correction. We calculated the mean standard deviation over all tracked fiducial markers of the same kind (Fig. 2c). Both, QBs and FSs yielded a mean standard deviation of their localization $\sigma_{\text {centroid }} \sim 5 \mathrm{~nm}$, while FSs showed a slightly higher stability during the recorded 2000 frames.

Next to the fluorescence properties, we characterized the contrast and size of the fiducial markers in electron microscopy. The TEM grid was unmounted from the fluorescence chamber, dried and transferred to a TEM. All fiducial markers appeared electron dense (Fig. 3a-c). The strongest contrast was observed for AuNPs and FSs (Fig. 3d, f), while QBs showed less contrast and more fluctuations in the signal (Fig. 3e). Due to the high TEM resolution, we could directly determine the circularity and size of individual fiducial markers. We found circularities of $0.91 \pm 0.03(n=21)$, $0.73 \pm 0.07(n=29)$ and $0.93 \pm 0.02(n=26)$ for AuNPs, QBs and FSs, respectively. Interestingly, the AuNPs showed the least variation (SD/mean) in size (23.0\%), while FSs showed a $31.8 \%$ variation and QBs up to $65.6 \%$ variation in 

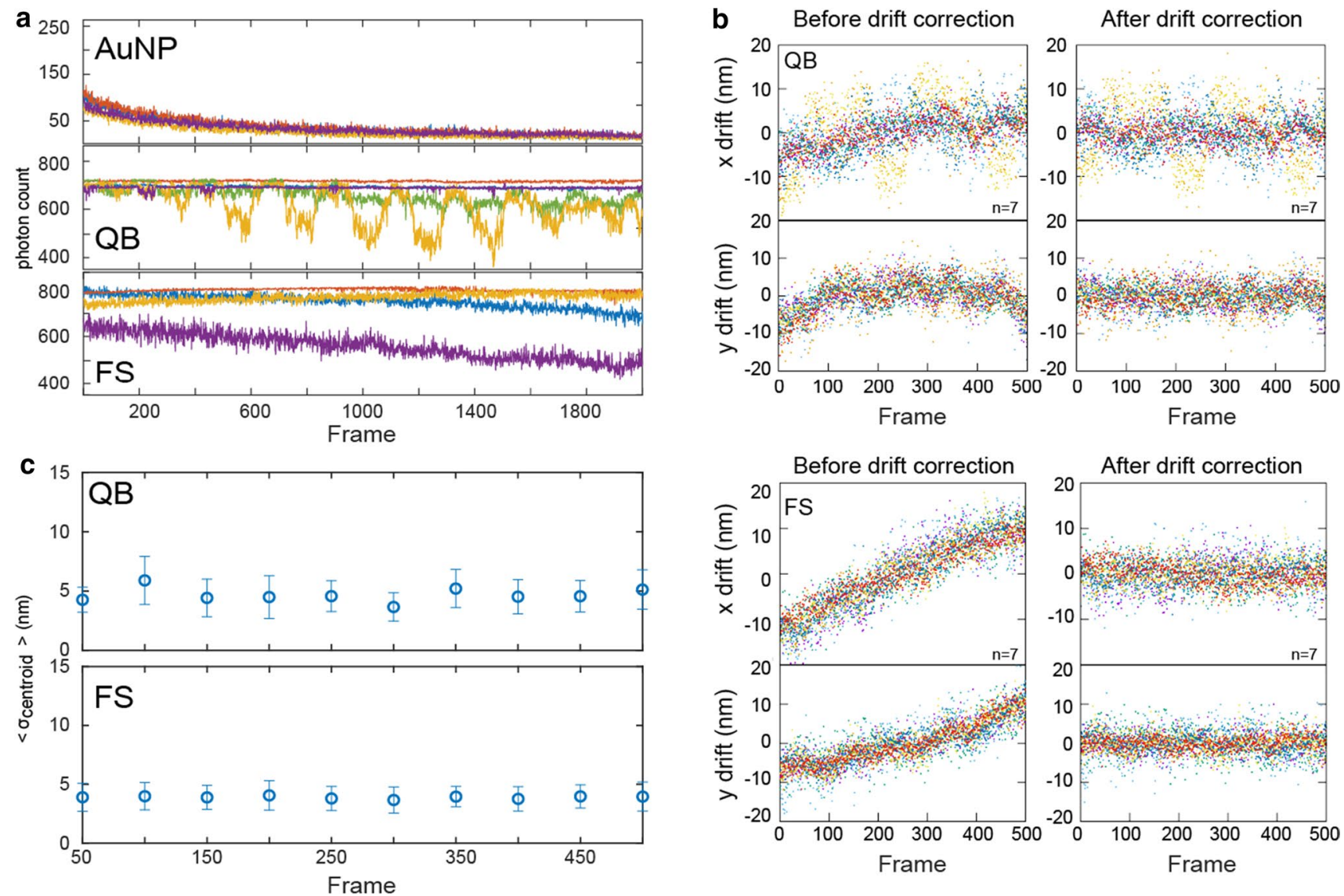

Fig. 2 Time stability of fiducials. a Intensity time traces $(n=4$, each) of Gold nanoparticles (AuNP), Quantum beads (QB) and FluoSpheres (FS). $\mathbf{b} \times$ and y positional drift for QBs and FSs, respectively, before and after drift correction. c Mean centroid localization accuracy of 7 individual QB and FS fiducial markers, time average over 50 frames

a

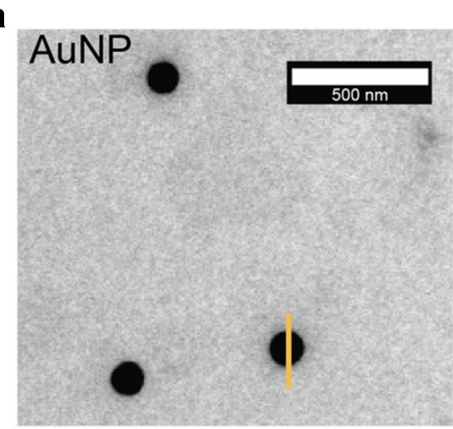

d

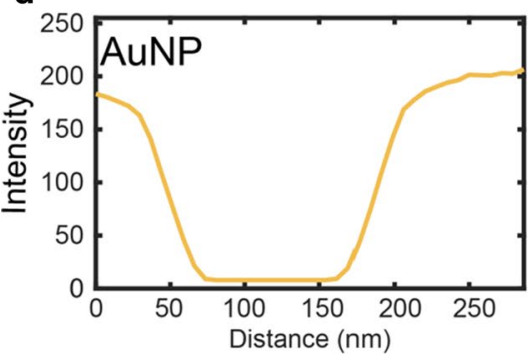

b

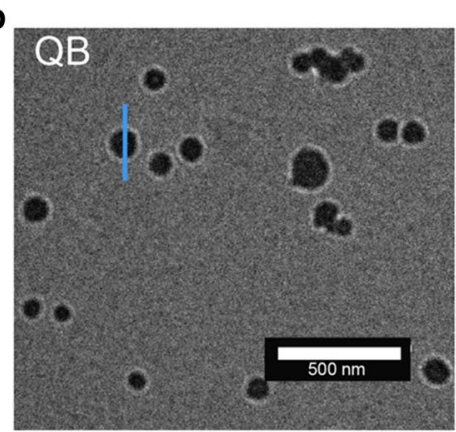

e

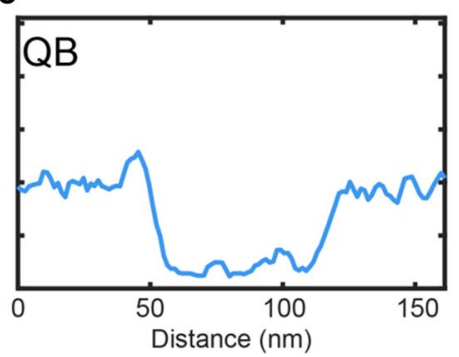

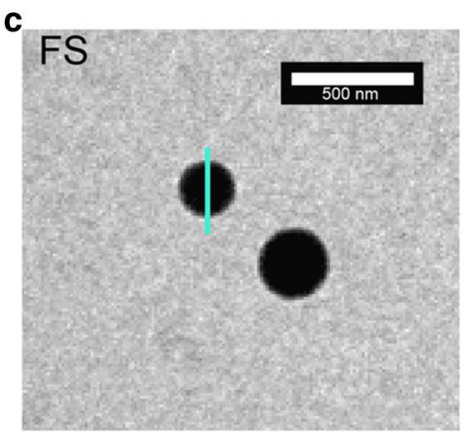

$\mathbf{f}$

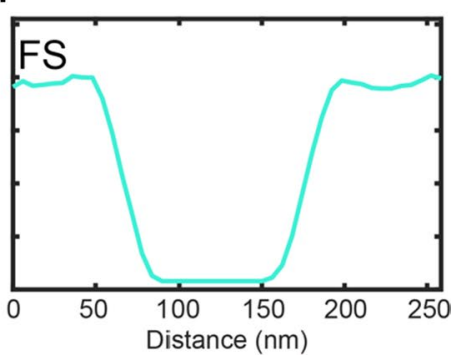

Fig. 3 Fiducial markers in TEM. Typical TEM micrographs of a AuNP b QBs and c FSs. d-f Intensity profiles along the corresponding lines in $(\mathbf{a}-\mathbf{c})$ 
size. Taking into account our observations from our fluorescence characterization and the TEM characterization, we conclude that spectrally shifted FSs appeared as most promising candidates for correlative super-resolution light and transmission electron microscopy experiments.

To characterize the accuracy of correlative experiments, we used FSs as landmarks and as a measure to quantify the overall deviation between both images. The schematic of the general workflow is given in Fig. 4a. To this extent, we imaged a high density of FSs on a carbon-coated TEM grid in buffer using single-molecule localization microscopy and determined the position of 16 markers in a single diffraction limited frame (Fig. 4b). The grid was subsequently dried and transferred for TEM for imaging. For image correlation, we developed a semi-automated MATLAB script. Briefly, fiducial pairs were manually selected for the corresponding field of view and their corresponding centroid positions were automatically determined with sub-pixel accuracy. The fluorescence image was scaled and rotated to match the electron microscopy image. Naturally, both imaging techniques have their limitations in the localization accuracy. The subsequent imaging, drying and transfer generates and additional correlation uncertainty, which we characterize in the following.

We calculate the overlay accuracy of an sCLEM image given a set of fiducials using the leave-one-out method, proposed by Kukulski and coworkers [21]. This means that one of the fiducial markers is used as ground truth sample, the remaining fiducial markers are used for automated alignment (Fig. 4a). After automated correlation, we measured the deviation of the ground truth fiducial between the EM and LM images, yielding a correlation localization accuracy $\sigma_{\mathrm{i}}=\sqrt{\sigma_{\mathrm{i}, \mathrm{x}}^{2}+\sigma_{\mathrm{i}, \mathrm{y}}^{2}}$, were i denotes the fiducial, $\sigma_{\mathrm{x}}=x_{\mathrm{EM}}-x_{\mathrm{LM}}$ and $\sigma_{\mathrm{y}}=y_{\mathrm{EM}}-y_{\mathrm{LM}}$. We repeated this procedure for each fiducial in the given set of fiducials per field of view and define the overlay accuracy as the average of the overlay error for all fiducials in the set $\sigma_{\mathrm{o}}=\frac{1}{\mathrm{~N}} \sum_{\mathrm{i}=1}^{\mathrm{N}} \sigma_{\mathrm{i}}$. For our given set of 16 fiducials, we derived an average overlay accuracy of $\sim 30$ $\mathrm{nm}$ given the permuted use of 15 fiducials for alignment (Fig. 4c). Yet, it remains unclear if this would be the best possible overlay accuracy, since some fiducial markers might have been displaced by drying or during imaging. Therefore, we analyzed subsets of fiducials from our set of 16 available fiducials. We picked 15 out of the 16 fiducials, and calculated for those an average overlay accuracy. Combinatorially, we thus have 16 subsets available for this evaluation and find that some subsets show a better average overlay accuracy according to the leave-one-out method than others (range 28 to $32 \mathrm{~nm}$ ). Using even smaller subsets, the spread of the average overlay accuracies further increased up to 6 to 97 $\mathrm{nm}$ for $\mathrm{n}=4$ fiducial sets out of the 16 available fiducials (Fig. 4c). Yet, the mean of the average overlay accuracies (blue line in Fig. 4c) steadily improved by the increase of fiducial markers available. Starting with a subset of 12 fiducials (\# 1-12 in Fig. 4b) showed a very similar trend in the overlay accuracy estimation. We further repeated this test by a triplicate on random 12 fiducials sets with similar trends though small variations on the mean overlay error (Additional file 1: Fig. S1). Unfortunately, a ground truth is in typical experiments not available to verify if the suggested overlay is more or less accurate. Therefore, we asked ourselves if there are obvious indications for a potential improvement of overlay accuracies. To show the effects of a single outlier on the overlay accuracy, we focused on 8 fiducial markers, which are likely to be in a typical field of view (\#1-8 Fig. 4b). We calculated for these 8 fiducials the resulting overlay accuracy and found a mean overlay accuracy $27 \mathrm{~nm}$. When we calculated from this data set now the combinatorial reduction for $7,6,5$, and 4 fiducial markers, we observed a bifurcation in the mean overlay accuracy. Upon a manual visual inspection of the fiducial markers, we identified a dimeric fiducial marker in the TEM image (Fig. 4c insets). After removal of this fiducial marker, the mean overlay accuracy was improved to $\approx 20 \mathrm{~nm}$. Moreover, this outlier could be unbiasedly removed with an automatic algorithm for outlier detection (see Methods). We repeated the combinatorial analysis of overlay accuracy 15 times from random $n=8$ fiducial sets and found that at least three sets present a clear bifurcation (Additional file 1: Fig. S2). Moreover, we found the mean overlay accuracy for 1000 random $n=8$ fiducial sets show a broad distribution from 18 to $50 \mathrm{~nm}$ depending on the subset and a mean overlay accuracy of $\approx 33 \mathrm{~nm}$ (Additional file 1: Fig. S3). We therefore suggest for an optimization of the overlay accuracy, a rigorous combinatorial analysis of the given fiducial markers to trace if a bifurcation of mean overlay accuracies occurs even among the fiducial markers, which indicates one or several non-optimal fiducial markers for the correlation of fluorescence and electron microscopy images. Such an automated approach is easy to implement and can be used prior to manual inspection for SCLEM or for subsequent estimation of overlay accuracy [22].

The diatom Thalassiosira pseudonana has an intricate nano-scale patterned biosilica cell wall [23]. Several proteins have been identified to be associated with the biosilica cell wall [24-28]. Current hypotheses suggest an underlying organic matrix, potentially generated by protein and other biomolecule self-assembly, which drives the nanoscale biosilica formation. Interestingly, dissolution of the silica of isolated biosilica cell walls, leaves behind an insoluble organic, nano-patterned matrix visible in $\operatorname{TEM}[28,29]$. In order to address the question if particular proteins can be associated with particular biomineral structures, SCLEM is a promising tool. Here, we studied the localization of the 


\section{a}

i) Imaging

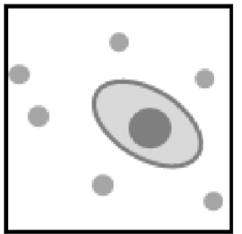

TEM ii) Manual selection of fiducial position followed by sub-pixel localization

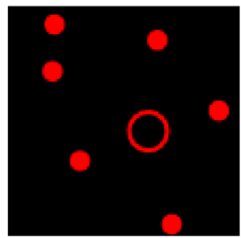

SMLM

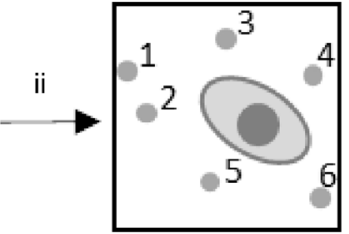

iii) Overlay for sCLEM

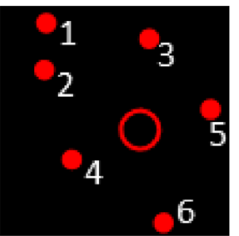

iv) Accuracy estimation with leave-one-out

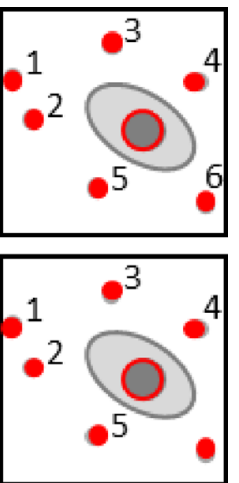

b
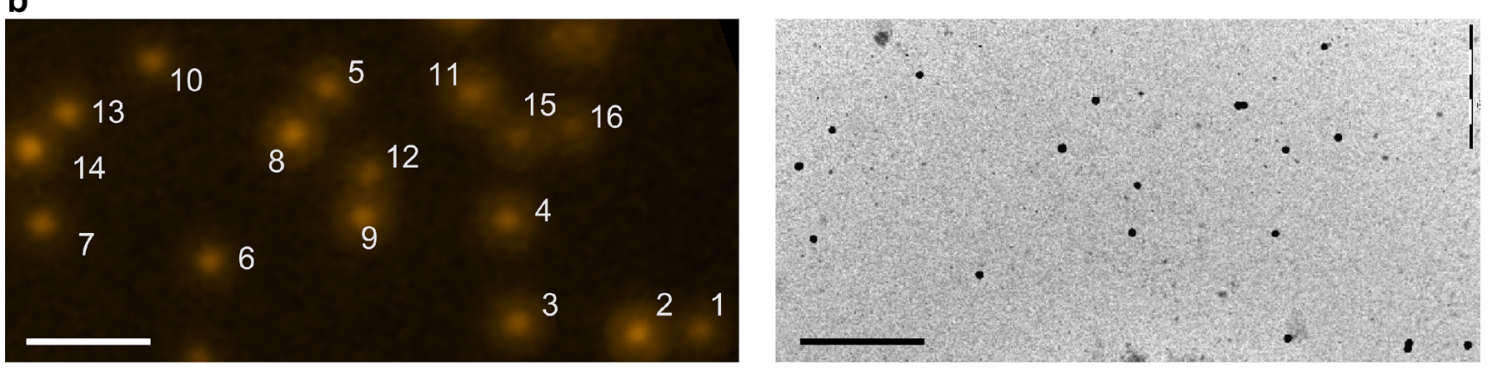

C
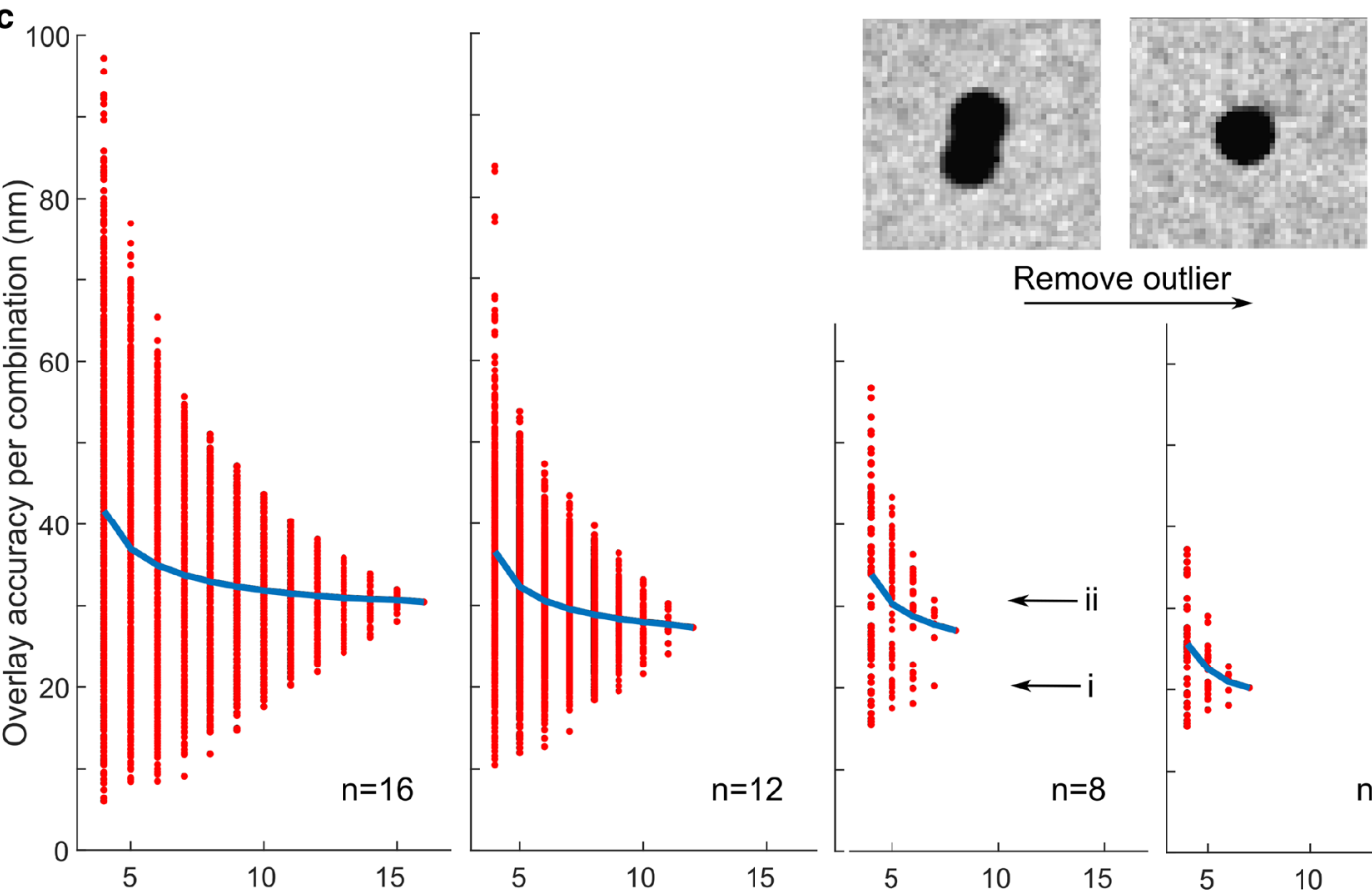

Remove outlier

Fiducial subset size

Fig. 4 Overlay accuracy determination. a Schematic of sCLEM workflow. The fluorescence image is transformed according to the corresponding position of fiducials on the EM image and overlaid onto the reference TEM image. $\mathbf{b}$ Fiducial markers (FSs, $n=16)$ in fluorescence and electron microscopy. Scale bars $2 \mu \mathrm{m}$. c Combinatorial analysis of leave-one-out overlay accuracy for all possible subsets of fiducials in an image. Leave-one-out overlay accuracy for each possible subset of the $n=16,12$ (from 1 to 12 in (b)) and 8 (from 1 to 8 in (b)) fiducials. $\mathrm{n}=7$ corresponds to the overlay accuracy of subsets in $\mathrm{n}=8$ after filtering out the dual-fiducial marker. Insets: TEM images of a dual fiducial and single fiducial marker, respectively 
protein Silicanin-1 within biosilica valves of the diatom Thalassiosira pseudonana. We generated a T. pseudonana strain in which the protein Silicanin-1 is tagged with Dendra2 at the N-terminus (Sin 1-Dendra2 ${ }^{\mathrm{N}}$ ). Biosilica valves were isolated from the cell culture [25], placed on the carbon-coated TEM grid with FSs as fiducial markers and imaged using photoactivatable light microscopy (Fig. 5a, b) [4, 30]. After drying, the same valves were imaged in a TEM and both images were correlated (Fig. $5 c, d$ ). The super-resolution image of $\operatorname{Sin} 1-D e n d r a 2^{\mathrm{N}}$ showed an average localization uncertainty of $33 \mathrm{~nm}$. The correlative overlay uncertainty was determined with the leave-one-out method to be $\sim 77 \mathrm{~nm}$ for seven fiducial markers (Fig. 5e). A systematic reduction of fiducial markers indicated that one fiducial marker (blue arrow) was particularly offset and after removal the overall uncertainty could be reduced to $\sim 49 \mathrm{~nm}$. Such a single fiducial marker could have been moved independently from the specimen and the rest of fiducials during washing or drying procedures, emphasizing that the permutated and reduced leave-one-out algorithm is important to increase the correlation. For $\operatorname{Sin} 1-D e n d r a 2^{\mathrm{N}}$, the correlative image did not reveal any obvious correlation indicating that $\operatorname{Sin} 1-D e n d r a 2^{\mathrm{N}}$ might be rather homogenously distributed in the center region of the valve. Further localization and inferred function within the biomineralization process remains to be determined.

\section{Conclusions}

Here, we compared three different fiducial markers, fluorophore-labeled gold nanoparticles (AuNPs), quantum beads (QBs) and spectrally shifted dark-red FluorSpheres (FSs) for correlative superresolution-transmission electron microscopy (sCLEM). For single-molecule localization microscopy, QBs and FSs were brighter, more homogenous in shape and more photostable than AuNPs. They allowed for an efficient drift correction during long imaging cycles with a mean localization uncertainty better than $5 \mathrm{~nm}$. All three markers appeared with sufficient contrast and regular circular shapes in EM, yet QBs showed the least contrast. Moreover, QBs needed an additional surface coating with poly-L-lysine to allow stronger immobilization, therefore we conclude that red-shifted FluoSpheres might be most suited for a correlation of SMLM and TEM using orange photoactivatable proteins. For overlays of SMLM and TEM images, we found typical overlay accuracies between 20 and $50 \mathrm{~nm}$, suggesting a high enough accuracy to place most proteins in a structural context in TEM images. Our systematic reduction of the available fiducial data sets further showed that bifurcations indicate single outlier fiducial markers and their elimination can increase the overlay accuracy up to 2-fold. We anticipate that correlative SMLM and TEM together with a careful evaluation of overlay accuracies can reveal structural correlates in the biomineralization process of diatoms or other single-cell organisms.
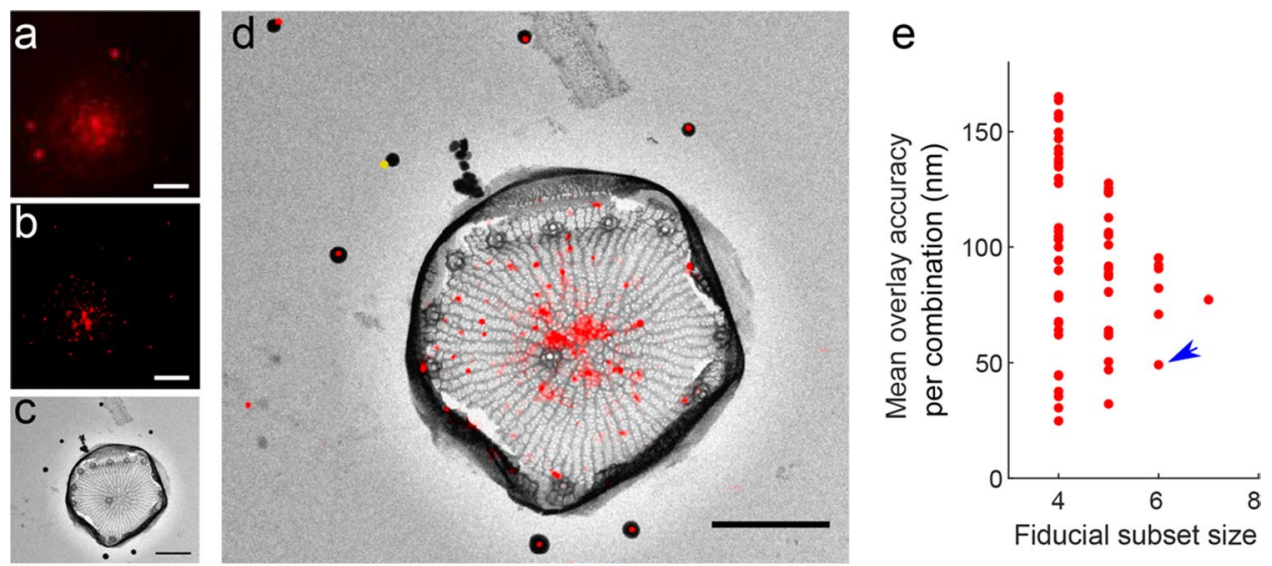

Fig. 5 SCLEM image of $\operatorname{Sin} 1-D e n d r a 2^{\mathrm{N}}$ on diatom valve $\mathbf{a}$ The diffraction limited and $\mathbf{b}$ reconstructed PALM images of Sin 1-Dendra $2^{\mathrm{N}}$. $\mathbf{c}$ TEM image of the same diatom valve. $\mathbf{d}$ sCLEM overlay image of the reconstructed PALM Sin1-Dendra $2^{\mathrm{N}}$ image and the TEM valve image. One fiducial marker (yellow) was omitted during alignment. e Overlay accuracy determination using the leave-one-out procedure and fiducial subsets. Removal of one fiducial (yellow in panel d) increased the overlay accuracy by $\sim 1.6$-fold (blue arrow). Scale bars $2 \mu \mathrm{m}$ 


\section{Materials and methods}

\subsection{Sample preparation}

Fiducial markers Quantum Dot Beads (120 nm, DNQ-N001, CD creative diagnostics), Alexa647 labeled gold nanoparticles $(100 \mathrm{~nm}, \mathrm{GFL}-100, \mathrm{CD}$ creative diagnostics) and dark red fluorescent $(660 / 680)$ FluoSpheres (200 nm, F8807, ThermoFisher scientific) were obtained commercially. Gold finder grids (G200F2-Au, Electron Microscopy Services) were coated with Formvar and carbon. For QBs, the TEM grids were additionally coated with Poly-L-Lysine $(0.1 \%$ solution, Sigma Aldrich) for $1 \mathrm{~h}$. FSs were diluted 1:200, AuNPs 1:15 and QBs 1:25,000 in milliQ water from their respective stock solutions to reach an approximate concentration of $2 \cdot 10^{10}$ particles $/ \mathrm{mL}$, sonicated (Elmasonic $\mathrm{S} 60 \mathrm{H}$ ) for 4 min to reduce aggregation, and deposited on the TEM grid surface for $20 \mathrm{~min}$. The grid was washed $3 \mathrm{x}$ in milliQ water and once in PBS and on a microscope slide with the sample surface facing up. The coverslip was placed on top after addition of $4 \mu$ PBS.

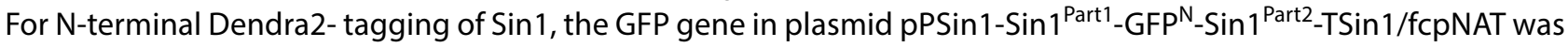
replaced by the Dendra2 encoding gene using the Kpnl and Notl restrictions sites flanking the GFP gene. The gene encoding for Dendra2 was amplified from pBluescript/Tp-fcp $p_{p+t}+$ Dendra2 by PCR using the sense primer $5^{\prime}-C G T C G C$ CTCGAGGATGGTACCGGTGGAATGAACACACCTGGA ATCAAC-3' and the antisense primer 5'-CCATCGTACGCAGCGGCC GCTCCCCAGACT TGTG-3'. The amplified Dendra2 gene was introduced into the Kpnl and Notl sites of the plasmid

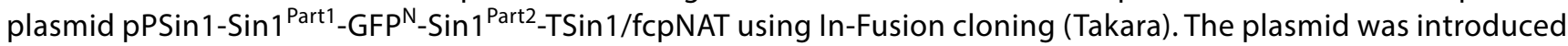
into T. pseudonana wild type cells using the biolistic PDS-1000/He particle delivery system (Bio-Rad) [31].

Diatom frustules were extracted from Thalassiosira pseudonana cells grown in Artificial Sea Water medium and harvested at $\sim 10^{6}$ cells $/ \mathrm{ml}$. Cells were pelleted by centrifugation at 3200xg for $10 \mathrm{~min}$ and frustules were isolated by incubating the cells in $2 \% \mathrm{SDS}, 100 \mathrm{mM}$ EDTA solution for $1 \mathrm{~h}$ at $50{ }^{\circ} \mathrm{C}$ and washing, repeated twice. Valves were separated from frustules with a $30 \mathrm{~s}$ tip sonicator. Coated TEM grids were incubated with poly-L-Lysine and $\sim 10 \mu \mathrm{L}$ sonicated silica shells were incubated for $30 \mathrm{~min}$. The grid was washed 3 times in milliQ and 1:50 diluted and presonicated FSs (1:50 from stock) were added for 20 min prior to imaging. The grid was washed again 3 times in milliQ and finally placed in PBS for imaging.

\subsection{Fluorescence spectra}

Fluorescence spectra of the three fiducial markers were obtained in a Horiba Duetta Bio spectrometer by diluting the stock solutions into PBS and the spectrum of Dendra2 from fpbase.org website.

\subsection{Super-resolution and electron microscopy imaging}

Microscope coverslips were cleaned by sonication for 15 min in Hellmanex $1 \%$, thorough washing with milliQ water, sonication for $15 \mathrm{~min}$ with $70 \%$ ethanol, and drying with nitrogen. Fiducials were excited with $20 \mathrm{~mW}$ end-of-fiber $561 \mathrm{~nm}$ excitation (12.6 mW at-the-objective power); PALM imaging was performed with $20 \mathrm{~mW}(12.6 \mathrm{~mW})$ and 50 $\mathrm{mW}(31.5 \mathrm{~mW}) 561 \mathrm{~nm}$ excitation. Images were recorded in near-TIRF condition with a 100x NA 1.49 objective on a Nikon microscope equipped for N-STORM and TIRF imaging using an Andor iXon Ultra 897 camera at 60 ms exposure and with a pre-gain of 3 and an EM gain of 300. We converted the analog to digital units (ADU) count obtained from the images to photon counts by the following equation, where $\mathrm{PE}=4.95$ (at pregain $3,17 \mathrm{MHz}, 16$ bit operation) is the photoelectrons per ADU and QE is the quantum efficiency of the detector at the wavelengths (0.95) and the offset $=+100$ (according to the manufacturer)

$$
\text { Photon count }=\frac{(\mathrm{ADU}-\mathrm{offset}) \cdot \mathrm{PE}}{\mathrm{EM} \text { gain } \cdot \mathrm{QE}}
$$

Reconstruction of the image was performed in Fiji [32] using the ThunderSTORM [20] plugin. After fluorescence imaging, the grid is removed from the coverslip by injecting milliQ water under the coverslip using a syringe and needle. The coverslip is thus floated in water to prevent the tear of the carbon/Formvar film of the TEM grid. It is then floated alternatingly on both sides on milliQ drops as described previously. The grid is then air-dried in ambient conditions in a grid box. No further contrast enhancement was done. TEM imaging was performed on a FEI Morgagni 268D with an SIS MegaView III camera at $80 \mathrm{keV}$. 


\section{4 sCLEM overlay and leave-one-out accuracy estimation}

The analysis was carried out in MATLAB R2018b. All scripts developed here are available upon request (see pseudocode in Additional file 1: Fig S5 for an overview of our code architecture). Briefly, we load LM and EM images and select landmarks manually using the cpselect function. We first picked 3 landmarks and then carried out a similarity transformation using fitgeotrans function based on this subset. This eases the process of picking the remaining fiducials in a guided fashion. Then, we stored the positions of all fiducials in the Field of View. The remaining procedure is automated using MATLAB scripts. First, we localize each fiducial with sub-pixel accuracy by thresholding and calculating the center of mass. When localizing the centroid using thresholding, we automatically pick a ROI that encloses each fiducial and uses 0.5 of the maximum intensity as the threshold for fluorescence and 0.2 for TEM images. A sensitivity analysis of overlay error as a function of thresholding shows negligible effects of varying the last parameters (Additional file 1: Fig. S4). We estimate the Overlay accuracy with the leave-one-out procedure which runs as follows: (1) Pick a combination of fiducials and leave one out of the set; (2) perform a transformation on the LM image (EM image fixed) and find the error in the distance of the excluded fiducial by locating it in both, EM and LM images, and subtracting the position; (3) repeat the process for each fiducial in the set. The overlay accuracy is defined as the mean value of previous measurements. To address the contribution of each fiducial on the overall overlay accuracy we used a combinatorial analysis of each possible subset of fiducials within an image of 16 landmarks. To exclude outliers, we measure the leave-one-out overlay error for each fiducial and remove landmarks with an error bigger than 1.5 IQR+Q3 (IQR: Inter Quartile Range, Q3:Third quartile) of the population, although, further methods can be used as well as MATLAB built-in functions [33].

Acknowledgements We acknowledge discussions with Nils Kröger (TU Dresden) and support by Jens Ehrig of the Molecular Imaging and Manipulation Facility and Thomas Kurth of the Electron Microscopy facility, two core facilities of the CMCB at TU Dresden. This study was supported by the Deutsche Forschungsgemeinschaft (DFG) within the Research Unit FOR2038 (SCHL1896/2-2 to M.S. and KR1853/6-2 to Nils Kröger) and the European commission through Erasmus+ (to C.A.Q.C.).

Authors' contributions MS and AF conceived the study. Material preparation and data collection was performed by AF, $\mathrm{CH}$ provided the diatom strain containing $\operatorname{Sin} 1-D e n d r a 2^{\mathrm{N}}$. Analysis script, analysis and figures were prepared by AF and CAQC, MS supervised the study and acquired funding. The first draft of the manuscript was written by AF and all authors commented on the manuscript and approved the final manuscript. All authors read and approved the final manuscript.

Funding Open Access funding enabled and organized by Projekt DEAL. This work was funded by the Deutsche Forschungsgemeinschaft (DFG) through Research Unit 2038 "NANOMEE" (DFG Grant SCHL1896/2-2 and KR1853/6-2 to Nils Kröger) and the European commission through Erasmus+ (to C.A.Q.C.).

Data availability Raw data is available upon request from the corresponding author.

Code availability Source code is available upon request from the corresponding author.

\section{Declarations}

Competing interests The authors declare that they have no competing interests

Open Access This article is licensed under a Creative Commons Attribution 4.0 International License, which permits use, sharing, adaptation, distribution and reproduction in any medium or format, as long as you give appropriate credit to the original author(s) and the source, provide a link to the Creative Commons licence, and indicate if changes were made. The images or other third party material in this article are included in the article's Creative Commons licence, unless indicated otherwise in a credit line to the material. If material is not included in the article's Creative Commons licence and your intended use is not permitted by statutory regulation or exceeds the permitted use, you will need to obtain permission directly from the copyright holder. To view a copy of this licence, visit http://creativecommons.org/licenses/by/4.0/.

\section{References}

1. de Boer P, Hoogenboom JP, Giepmans BNG. Correlated light and electron microscopy: ultrastructure lights up! Nat Methods. 2015;12:503-13. https://doi.org/10.1038/nmeth.3400.

2. Müller-Reichert T, Verkade P. (2017) Correlative Light and Electron Microscopy III, Vol. 140.

3. Deschout H, Zanacchi FC, Mlodzianoski M, Diaspro A, Bewersdorf J, Hess ST, Braeckmans K. Precisely and accurately localizing single emitters in fluorescence microscopy. Nat Methods. 2014;11:253-66. https://doi.org/10.1038/nmeth.2843.

4. Betzig E, Patterson GH, Sougrat R, Lindwasser OW, Olenych S, Bonifacino JS, Davidson MW, Lippincott-Schwartz J, Hess HF. Imaging intracellular fluorescent proteins at nanometer resolution. Science. 2006;313:1642-5. https://doi.org/10.1126/science.1127344. 
5. Rust MJ, Bates M, Zhuang X. Sub-diffraction-limit imaging by stochastic optical reconstruction microscopy (STORM). Nat Methods. 2006;3:7935. https://doi.org/10.1038/nmeth929.

6. Heilemann M, Van De Linde S, Schüttpelz M, Kasper R, Seefeldt B, Mukherjee A, Tinnefeld P, Sauer M. Subdiffraction-resolution fluorescence imaging with conventional fluorescent probes. Angew Chemie - Int Ed. 2008;47:6172-6. https://doi.org/10.1002/anie.200802376.

7. Kukulski W, Schorb M, Welsch S, Picco A, Kaksonen M, Briggs JAG. (2012) Precise, Correlated Fluorescence Microscopy and Electron Tomography of Lowicryl Sections Using Fluorescent Fiducial Markers. In: Methods in Cell Biology. Academic Press Inc., pp $235-257$.

8. Sosinsky GE, Giepmans BNG, Deerinck TJ, Gaietta GM, Ellisman MH. (2007) Markers for Correlated Light and Electron Microscopy. Methods Cell Biol. 2007:575-591.

9. van Hest JJHA, Agronskaia AV, Fokkema J, Montanarella F, Gregorio Puig A, de Mello Donega C, Meijerink A, Blab GA, Gerritsen HC. Towards robust and versatile single nanoparticle fiducial markers for correlative light and electron microscopy. J Microsc. 2019;274:13-22. https://doi. org/10.1111/jmi.12778.

10. Schellenberger P, Kaufmann R, Siebert CA, Hagen C, Wodrich H, Grünewald K. High-precision correlative fluorescence and electron cryo microscopy using two independent alignment markers. Ultramicroscopy. 2014;143:41-51. https://doi.org/10.1016/j.ultramic.2013.10.011.

11. van Elsland DM, Pujals S, Bakkum T, Bos E, Oikonomeas-Koppasis N, Berlin I, Neefjes J, Meijer AH, Koster AJ, Albertazzi L, van Kasteren SI. Ultrastructural imaging of Salmonella-host interactions using super-resolution correlative light-electron microscopy of bioorthogonal pathogens. ChemBioChem. 2018;19:1766-70. https://doi.org/10.1002/cbic.201800230.

12. Haruta T, Hasumi K, Ikeda Y, Konyuba Y, Fukuda T, Nishioka H. Local surface plasmon resonance of gold nanoparticles as a correlative light and electron microscopy (CLEM) tag for biological samples. Microscopy. 2019;68:467-70. https://doi.org/10.1093/jmicro/dfz031.

13. Fokkema J, Fermie J, Liv N, van den Heuvel DJ, Konings TOM, Blab GA, Meijerink A, Klumperman J, Gerritsen HC. Fluorescently labelled silica coated gold nanoparticles as fiducial markers for correlative light and electron Microscopy. Sci Rep. 2018;8:13625. https://doi.org/10.1038/ s41598-018-31836-1.

14. Killingsworth MC, Bobryshev YV. (2016) Correlative light- and electron microscopy using quantum dot nanoparticles. J Vis Exp 2016:e54307. https://doi.org/10.3791/54307.

15. Masich S, Östberg T, Norlén L, Shupliakov O, Daneholt B. A procedure to deposit fiducial markers on vitreous cryo-sections for cellular tomography. J Struct Biol. 2006;156:461-8. https://doi.org/10.1016/j.jsb.2006.05.010.

16. Balinovic A, Albrecht $D$, Endesfelder U. Spectrally red-shifted fluorescent fiducial markers for optimal drift correction in localization microscopy. J Phys D Appl Phys. 2019;51:204002. https://doi.org/10.1088/1361-6463/ab0862.

17. Wang S, Moffitt JR, Dempsey GT, Xie XS, Zhuang X. Characterization and development of photoactivatable fluorescent proteins for singlemolecule-based superresolution imaging. Proc Natl Acad Sci U S A. 2014;111:8452-7. https://doi.org/10.1073/pnas.1406593111.

18. Tokunaga M, Imamoto N, Sakata-Sogawa K. Highly inclined thin illumination enables clear single-molecule imaging in cells. Nat Methods. 2008;5:159-61. https://doi.org/10.1038/nmeth1171.

19. Hohng S, Ha T. Near-complete suppression of quantum dot blinking in ambient conditions. J Am Chem Soc. 2004;126:1324-5. https://doi. org/10.1021/ja039686w.

20. Ovesný M, Křížek P, Borkovec J, Švindrych Z, Hagen GM. ThunderSTORM: A comprehensive Image J plug-in for PALM and STORM data analysis and super-resolution imaging. Bioinformatics. 2014;30:2389-90. https://doi.org/10.1093/bioinformatics/btu202.

21. Kukulski W, Schorb M, Welsch S, Picco A, Kaksonen M, Briggs JAG. Correlated fluorescence and 3D electron microscopy with high sensitivity and spatial precision. J Cell Biol. 2011;192:111-9. https://doi.org/10.1083/jcb.201009037.

22. Yang JE, Larson MR, Sibert BS, Shrum S. (2020) CorRelator: An interactive and flexible toolkit for high-precision cryo-correlative light 1 and electron microscopy 23. bioRxiv 2020.08.06.240481. https://doi.org/10.1101/2020.08.06.240481.

23. Kröger N, Poulsen N. Diatoms - from cell wall biogenesis to nanotechnology. Annu Rev Genet. 2008;42:83-107. https://doi.org/10.1146/annur ev.genet.41.110306.130109.

24. Kröger N, Lorenz S, Brunner E, Sumper M. Self-assembly of highly phosphorylated silaffins and their function in biosilica morphogenesis. Science. 2002;298:584-6. https://doi.org/10.1126/science.1076221.

25. Kotzsch A, Gröger P, Pawolski D, Bomans PHH, Sommerdijk NAJM, Schlierf M, Kröger N. Silicanin-1 is a conserved diatom membrane protein involved in silica biomineralization. BMC Biol. 2017;15:65. https://doi.org/10.1186/s12915-017-0400-8.

26. Kirkham AR, Richthammer P, Schmidt K, Wustmann M, Maeda Y, Hedrich R, Brunner E, Tanaka T, Van Pée KH, Falciatore A, Mock T. A role for the cell-wall protein silacidin in cell size of the diatom Thalassiosira pseudonana. ISME J. 2017;1 1:2452-64. https://doi.org/10.1038/ ismej.2017.100.

27. Tesson B, Lerch SJL, Hildebrand M. (2017) Characterization of a new protein family associated with the silica deposition vesicle membrane enables genetic manipulation of diatom silica. Sci Rep 7:. https://doi.org/10.1038/s41598-017-13613-8.

28. Scheffel A, Poulsen N, Shian S, Kröger N. Nanopatterned protein microrings from a diatom that direct silica morphogenesis. Proc Natl Acad Sci U S A. 2011;108:3175-80. https://doi.org/10.1073/pnas.1012842108.

29. Heintze C, Formanek P, Pohl D, Hauptstein J, Rellinghaus B, Kröger N. An intimate view into the silica deposition vesicles of diatoms. BMC Mater. 2020;2:11. https://doi.org/10.1186/s42833-020-00017-8.

30. Gröger P, Poulsen N, Klemm J, Kröger N, Schlierf M. Establishing super-resolution imaging for proteins in diatom biosilica. Sci Rep. 2016;6:1-8. https://doi.org/10.1038/srep36824.

31. Poulsen N, Chesley PM, Kröger N. Molecular genetic manipulation of the diatom Thalassiosira pseudonana (Bacillariophyceae). J Phycol. 2006;42:1059-65. https://doi.org/10.1111/j.1529-8817.2006.00269.x.

32. Schindelin J, Arganda-Carreras I, Frise E, Kaynig V, Longair M, Pietzsch T, Preibisch S, Rueden C, Saalfeld S, Schmid B, Tinevez JY, White DJ, Hartenstein V, Eliceiri K, Tomancak P, Cardona A. Fiji: An open-source platform for biological-image analysis. Nat Methods. 2012;9:676-82. https://doi.org/10.1038/nmeth.2019.

33. Rousseeuw PJ, Hubert M. Robust statistics for outlier detection. WIREs Data Min Knowl Discov. 2011;1:73-9. https://doi.org/10.1002/widm.2.

Publisher's note Springer Nature remains neutral with regard to jurisdictional claims in published maps and institutional affiliations. 